\title{
Redistribution of Mobile Surface Charges of an Oil Droplet in Water in Applied Electric Field
}

\author{
Mengqi Li and Dongqing Li* \\ Department of Mechanical and Mechatronics Engineering, University of Waterloo, \\ Waterloo, Ontario, Canada N2L 3G1
}

*Corresponding author, Address: 200 University Ave. West, Waterloo, Ontario, N2L 3G1

Email: dongqing@uwaterloo.ca (D. Li)

\section{Statement}

The final publication is available at Elsevier via http://dx.doi.org/10.1016/j.cis.2016.08.006. () 2016. This manuscript version is made available under the CC-BY-NC-ND 4.0 license http://creativecommons.org/licenses/by-nc-nd/4.0/ 


\begin{abstract}
Most researches on oil droplets immersed in aqueous solutions assume that the surface charges of oil droplets are, similar to that of solid particles, immobile and distributed uniformly under externally applied electric field. However, the surface charges at the liquid-liquid interface are mobile and will redistribute in response to the electric field. This paper studies the redistribution of surface charges on an oil droplet under the influence of the external electrical field. Analytical expressions of the local zeta potential on the surface of an oil droplet after the charge redistribution in a uniform electrical field were derived. The effects of the initial zeta potential, droplet radius and strength of applied electric field on the surface charge redistribution were studied. In analogy to the mobile surface charges, the redistribution of $\mathrm{Al}_{2} \mathrm{O}_{3}$-passivated aluminum nanoparticles on the oil droplet surface was observed under applied electrical field. Experimental results showed that these nanoparticles moved and accumulated towards one side of the oil droplet in response to the applied electric field. The redistribution of the nanoparticles is in qualitative agreement with the redistribution model of the mobile surface charges developed in this work.
\end{abstract}

Key Words: mobile surface charges, liquid-fluid interface, redistribution of surface charges, local zeta potential, electric field 


\section{Contents}

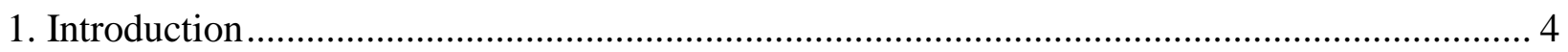

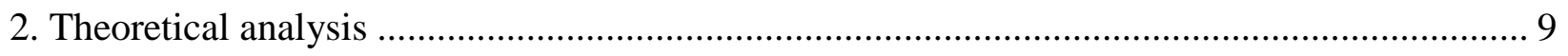

2.1 Electrical field around oil droplet .................................................................... 10

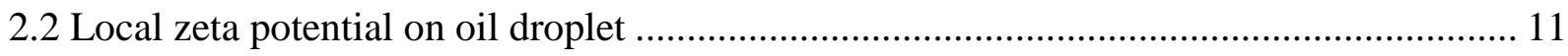

2.3 Local surface charge density on oil droplet ............................................................ 12

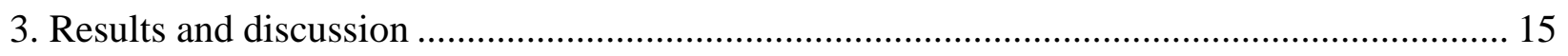

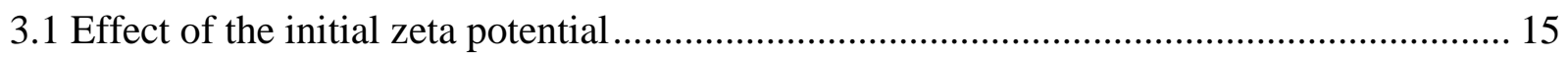

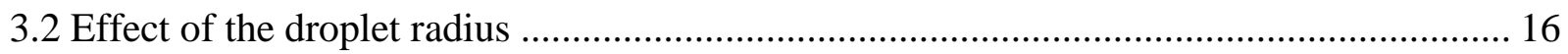

3.3 Effect of the applied electric field........................................................................ 16

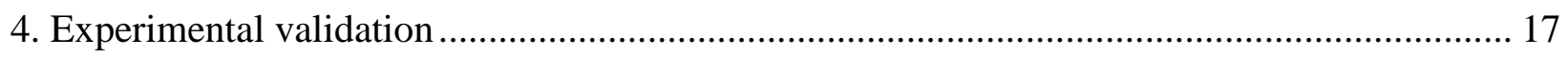

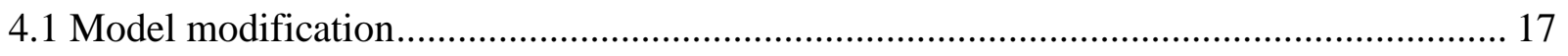

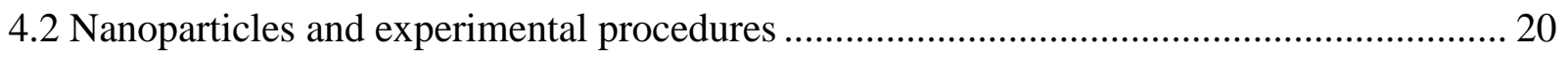

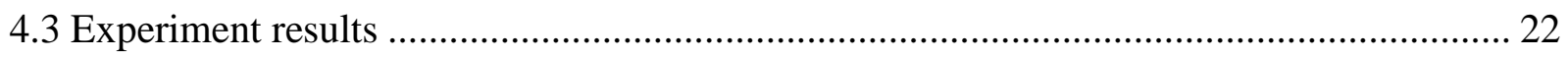

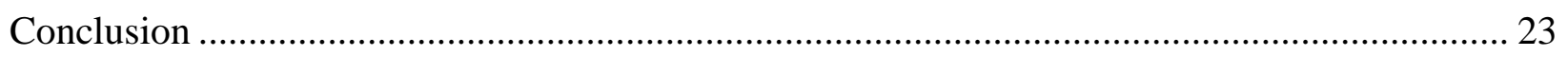

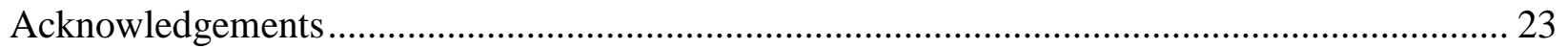

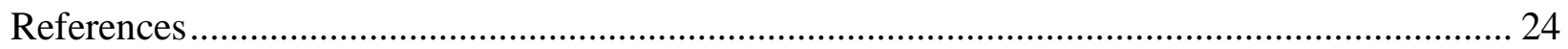




\section{Introduction}

Electrostatic surface charges on solid particles and oil droplets are very important in various applications involving electroosmosis and electrophoresis [1-9]. It is well-known that the surface charges at the solid-liquid interfaces are immobile. However, the situation for liquid-liquid interfaces is different because of the mobility of the liquid-liquid interfaces. For example, the most widely accepted explanation is that the charges at the oil-water interfaces originate from the selective adsorption of hydroxide ions $\left(\mathrm{OH}^{-}\right)$[10-15]. For an oil droplet immersed in water, the hydroxide ions will be adsorbed to the surface of the oil droplet and make the oil-water interface negatively charged. Another possibility is that the existence of some ionic surface-active impurities in oil phase may be responsible for creating the charges at the oil-water interfaces[16,17]. While the mechanism of charge creation on the liquid-liquid interfaces is still under discussion, the mobility of the surface charges at the liquid-liquid interfaces is widely recognized.

The mobile surface charges at the liquid-liquid interface were considered in the investigation of multiphase electroosmotic flow (EOF). It is well-known that all surfaces acquire electrostatic charges when they are in contact with an aqueous electrolyte solution. The surface charges in turn attract the counter-ions in the bulk liquid to the region close to the surface, forming the electric double layer (EDL). Under a tangentially applied electrical field, the excess counter-ions in the double layer region will move, resulting in a bulk liquid motion via viscous effect. This is known as the electroosmotic flow (EOF). In 2005, Gao et al. [18] analyzed the transient twoliquid phase EOF by considering the effect of the free surface charges at the liquid-liquid interface. The transient EOF of the two-liquid phase system was calculated by a model which considered a force at the interface generated by the electrokinetic motion of the free surface charges. In 2006, Lee et al. [19] conducted numerical simulations of the EOF over a liquid-liquid interface based on three different models: viscous model [20], electrical double layer (EDL) model [21], and EDL plus surface charges (EDL+SC) model. It was found that the EDL+SC model is the best to evaluate the multiphase EOF involving a liquid-fluid interface, as the predicted results of EDL+SC model and the experimental results agreed well with each other. The EDL+SC model considered the combined influence of the EDL and the mobile surface charges on the motion of liquid-liquid interface. For example, in a uniform DC electric field, if a 
liquid-liquid interface is negatively charged, the negative charges at the interface moves towards the anode and the counter-ions in the EDL moves in the opposite direction. Both of these movements contribute to the motion of the interface. Later, Lee et al. [22] experimentally studied the EOF at liquid-air interface. The experimental results indicated that the electroosmotic velocity of the liquid at the interface was significant lower than that in the bulk liquid, which also agreed with the EDL+SC model mentioned above. Based on this model, Movahed et al. [23] studied the EOF of a stream of aqueous electrolyte solution surrounded by oil. In addition, Gao et al. [24] theoretically and experimentally investigated two-liquid phase EOF with pressure gradient effect. The combined effects of the mobile surface charges, electroosmosis and pressure gradient at the interface were taken into consideration in their model. The comparison between the theoretically predicted results and the experimental results showed very good agreement, confirming the mobility of surface charges at the interface. In 2010, Li et al. [25] studied the time-dependent three-fluid phase EOF by accounting the mobile charges at the liquid-liquid interface. In their model, the effect of the shear force at the interface generated by the electrokinetic motion of mobile surface charges was taken into consideration. Qian et al. [26], Choi et al. [27], and Ray et al. [28] studied the free surface EOF. It was demonstrated that the zeta potential of the free surface determined the motion of the liquid.

The redistribution of surface charges at liquid-liquid interface has also been pointed out in a study of the attachment of nanoparticles at liquid-liquid interface. By using X-ray photoelectron spectroscopy to detect the distribution of nanoparticles carrying negative charges near the negatively charged air-water interface, Redondo et al. [29] found that the more negative the charged nanoparticle, the closer it approaches the air-water interface. Their explanation for this is that nanoparticles with stronger negative charges will induced surface charge redistribution of the air-water interface and enable the nanoparticles to get closer to the interface. Furthermore, $\mathrm{Xu}$ et al. [30] observed that when a particle-coated water droplet gets close to a flat oil-water interface laden with particles, the particles on the flat interface will be repelled away from the closest approaching point due to the electrostatic interaction of the two interfaces, which also proves that the external electric field will make the surface charges at liquid-liquid interfaces redistribute. 
It is not difficult to understand that the electrostatic charges on the surface of an oil droplet are also mobile, and the distribution of these mobile surface charges will be changed under influence of external electric field. Such redistribution of surface charges will have impacts in many applications. For example, the surface charges on oil droplets will migrate in electric field, which will affect the coalescence of the oil droplets. Ichikawa et al. [31] found that the rate of the coalescence of oil droplets can be increased significantly with the effect of the redistributed surface charges on the oil droplets. Another easily understandable example is the electrophoresis of oil droplets in aqueous solutions, where the redistribution of surface charges will significantly affect the electrophoretic force acting on the droplets and hence the electrophoretic motion of the oil droplets. However, most of the studies on oil droplets immersed in aqueous solutions assumed that the surface charges were immobile and uniformly distributed in the presence of electric field. Such a treatment would cause the underestimate or overestimate of the surface charge from the measured electrophoretic mobility data of oil droplets. Therefore, fundamental research on the redistribution of surface charges of the oil droplet is necessary.

Theoretical studies on the surface charge redistribution under externally applied electric field were reported in some papers [32-39]. In summary, two models were derived based on different considerations. First, the expression of local electrostatic potential on oil droplet in externally applied electric field was derived by Ichikawa [34] relying on the relationship between the electrical potential $(\varphi)$ on the surface of the oil droplet and the surface charge density $(\sigma)$ :

$$
\sigma=-\left[\varepsilon_{m} \frac{\partial \varphi}{\partial n}\right]_{\text {surface }}
$$

where $\varepsilon_{m}$ is the dielectric constant of the surrounding electrolyte and $n$ is the normal to the surface.

The electrical potential distribution around a charged oil droplet can be obtained by solving the Poisson-Boltzmann equation. The surface charge density is proportional to the concentration difference between cations and anions absorbed at the interface, and the distribution of these ions is given by the Boltzmann distribution under electrical field potential arising from the local zeta potential and the externally applied electric field. Substituting these conditions into Eq. (1), the surface electrical potential $\left(\varphi_{s}\right)$ arising from the redistributions of adsorbed surface ions in a spherical coordinates system can be obtained: 


$$
\varphi_{s}=\zeta_{0}+\frac{z e \zeta_{0}\left(s_{+, 0}+s_{-, 0}\right)}{\kappa T\left(s_{+, 0}-s_{-, 0}\right)+z e \zeta_{0}\left(s_{+, 0}+s_{-, 0}\right)} E_{\infty} a \cos \theta
$$

where $E_{\infty}$ is the externally applied electric field, $a$ is the radius of the oil droplet, $z$ is the valence of the ions, $e$ is the elementary charge, $\kappa T$ is the thermal energy at temperature $T, S_{ \pm, 0}$ are the positive and negative ions surface number densities, respectively.

The derivation shown above was conducted under two assumptions: 1) Positive ions and negative ions coexist on the surface of oil droplets; 2) The total numbers of positive and negative ions remain unchanged before and after applying electric field. Therefore, based on this model, positive surface charges and negative surface charges can be induced to reside separately on different sides of oil droplets under electric field. However, when we consider about the charging mechanism of the oil droplet surfaces, it is easy to find that these assumptions are not practical. First, in electrical neutral solution $(\mathrm{pH}=7)$, the oil droplet will carry negative charges by adsorbing hydroxide ions or hydrolyzing ionic surface-active impurities, and only negative ions exist at the interface. Second, after the redistribution of the surface ions, if the concentration of certain type of ions at some places of the oil droplet surface is reduced to a value below the initial equilibrium condition, new surface charges will be generated at this part of the surface; therefore, the total number of surface ions will change.

The second model was derived by considering the balance between the flux of electrophoretic migration of charged species and that of back diffusion at stable state. In a spherical coordinate system, the balance can be obtained $[35,38]$ :

$$
m E_{e} C(\theta)=D \nabla C(\theta)
$$

where $m$ and $D$ are the electrophoretic mobility and diffusion coefficient of the charged species on the surface, $E_{e}$ is the local electric field, $C(\theta)$ is the surface concentration of the charged species.

The above equation can be solved with the boundary condition that the total numbers of the charged species on the surface remain the same before and after applying electric field. The surface charge density $(\sigma(\theta))$ can be obtained: 


$$
\sigma(\theta)=\frac{3 \pi}{4} \frac{a^{2} E_{\infty}}{\delta \psi} \frac{\sigma_{0}}{\sinh \left(\frac{3 \pi a^{2} E_{\infty}}{4 \delta \psi}\right)} \varepsilon_{m}^{-\frac{3 \pi a^{2} E_{\infty} \cos \theta}{4 \delta \psi}}
$$

where $\sigma_{0}$ is the initial surface charge density, $\delta$ is the average diameter of a charged complex, $\psi$ is the thermal potential. In this model, the boundary condition of constant number of mobile surface charges is also used, which generally is not valid for most liquid-liquid interfaces. This limits its application only for evaluating the redistribution of charged molecules on cells [35-39].

Few experiments of visualizing the surface charges have been reported in literature. The existing measurement methods of visualizing the distribution of charges at the interface can be divided into three groups. First, the charged fluorescent dyes were used to show the concentration of ions at the interface. Through measuring the fluorescent intensity at the interface, the surface charge density can be inferred qualitatively. According to the microscope used to detect the fluorescent intensity at the interface, the charged dye method can be divided into two categories: evanescent wave excitation method [40,41] and confocal scanning method [42,43]. However, these methods are generally used to detect the zeta potential of solid-liquid interfaces and monolayers on them, respectively. Second, the X-ray reflectivity measurements can reflect the structure of the liquidliquid interface. By using the X-ray reflectivity method, the electron distribution of the interface can be evaluated [44-46]. But this method is hard to control, and needs a complex system to generate X-ray and detect the reflective wave from the interface. Finally, the capacitance method was used to measure the zeta potential of the liquid-liquid interface in some papers [47-49]. However this method is easily affected by many other parameters, for example, the types of ionic species; therefore, the measured zeta potential was often in disagreement with the classical theory. Due to the limitations of the existing detection methods, the redistribution of mobile surface charges on oil droplets was only proved indirectly. For example, through measuring the EOF velocity at oil-water interfaces, the mobility of surface charges was proved [19]. The experimentally observed phenomenon of the rapid demulsification of oil-in-water emulsion under electric field can confirm that the external electric field will lead to the redistribution of surface charges on oil droplets and speed up the coalescence of oil droplets [31]. Up to date, no experimental research has been conducted to quantitatively study the redistribution of surface charges on oil droplet under the influence of externally applied electric field. 
In order to provide a better understanding of the electrokinetically-driven redistribution of mobile surface charges on liquid-liquid interface, this paper presents a fundamental investigation of the surface charge redistribution of an oil droplet suspended in an aqueous solution under the influence of externally applied electric field. First, surface charge redistribution model for a spherical surface was derived to describe the redistribution of the surface charges in uniform DC electric field. Then, the redistribution of zeta potential along the surface of an oil droplet can be calculated by using the model. The effects of the initial zeta potential of the droplet, the external electric field and the droplet size on the surface area with the redistributed charges and the local zeta potential were studied. Finally, experiments were carried out in an effort to visualize the charge redistribution. $\mathrm{Al}_{2} \mathrm{O}_{3}$-passivated aluminum nanoparticles were placed on the surface of the oil droplet to simulate the mobile charged molecules. The motion and redistribution of these nanoparticles were observed under externally applied electric field.

\section{Theoretical analysis}

Consider a spherical oil droplet surrounded by an aqueous electrolyte solution, as illustrated in Figure 1. Without loss of generality, let us assume the oil-water interface carries negative charges. The surface charges are uniformly distributed over the entire surface of the droplet if no external electrical field exists. When electric field is applied, the mobile negative charges on the droplet surface moves towards the anode or the high potential side of the electric field. With the motion of the mobile charges on the oil droplet, the quantity of charges on the other side of the oil droplet facing the cathode decreases, and the oil-water interface on this side is recharged to reach the local equilibrium under a given $\mathrm{pH}$ value, as shown in Figure 1. Then, the newly charged molecules will be further attracted to the anode, and the side of the oil droplet facing the cathode will keep generating new charges. This process will continue until the accumulated charges on the anode side reach the equilibrium state. At the equilibrium state, the local concentration of the redistributed surface charges is determined by the balance between the local electric field generated by the accumulated surface charges and the local externally applied electric field along the surface of the droplet. The minimum local zeta potential stays at the pole facing the cathode of the electric field, and should equal to the initial zeta potential of the oil droplet before the electric field is applied. 
To analyze the redistribution of the mobile surface charges, a spherical coordinate system is used where the origin point, $O$, is located at the center of the droplet and the direction of the $\mathrm{x}$-axis is the same as that of the applied electric field, as shown in Figure 2. The radius of the oil droplet is denoted as $a$.

\subsection{Electrical field around oil droplet}

For an oil droplet immersed in the aqueous solution, under a uniform externally applied electrical field $\phi=-E_{\infty} x$ or $\phi=-E_{\infty} r \cos \theta$ in the spherical coordinates system, the electrical field in the aqueous solution can be calculated with the well-known Laplace's equation:

$$
\nabla^{2} \phi=0
$$

In the spherical coordinate system whose origin stays in the center of the droplet, the Laplace's Equation can be extended into this form:

$$
\nabla^{2} \phi=\frac{1}{r^{2}} \frac{\partial}{\partial r}\left(r^{2} \frac{\partial \phi}{\partial r}\right)+\frac{1}{r^{2} \sin \theta} \frac{\partial}{\partial \theta}\left(\sin \theta \frac{\partial \phi}{\partial \theta}\right)=0
$$

$\phi$ has finite value at the origin. And the electrical field is equal to the externally applied electrical field when $r$ goes to infinity, i.e., $r \rightarrow \infty, \phi=-E_{\infty} r \cos \theta$. Therefore, the general solution of electrical potential in the aqueous solution $\phi$ by separation of variables is[50]:

$$
\phi=\sum_{k=0}^{\infty} A_{k} r^{-(k+1)} P_{k}(\cos \theta)
$$

where $A_{k}$ is constant and $P_{k}(\cos \theta)$ is the Legendre polynomial.

Under the externally applied electrical field, the mobile negative charges on the oil droplet move and get redistributed. With the driving of the electrical field and the redistributed surface charges, the positive ions in the electrolyte are delivered into the electric double layer (EDL) near the oil droplet. And the positive ions inside EDL get redistributed in response to the redistributed surface charges. Simultaneously, the redistributed screening cloud of ions inside the EDL of the oil droplet expels the electric field lines and the current going from the bulk liquid to the EDL gets reduced [51]. At equilibrium state, all of the electric field lines get expelled, which yields the no-flux boundary condition on the outer surface of the EDL region:

$$
\left(\frac{\partial \phi}{\partial r}\right)_{r=a+1 / \kappa}=0
$$

where $\kappa$ is the Debye-Huckel parameter:

$$
\kappa=1 / \sqrt{\frac{\varepsilon_{m} \varepsilon_{0} K_{b} T}{2 n_{\infty}(z e)^{2}}}
$$


where $z$ is the valence of ions, $n_{\infty}$ is the bulk ionic concentration, $e$ is the elementary charge, $K_{b}$ is the Boltzmann constant, $T$ is the absolute temperature. $\varepsilon_{0}$ and $\varepsilon_{m}$ are the dielectric permittivity of vacuum and the electrolyte, respectively.

For thin EDL, $a \gg 1 / \kappa$, Eq. (8) can be approximated to:

$$
\left(\frac{\partial \phi}{\partial r}\right)_{r=a}=0
$$

Applying this boundary condition (Eq. (10)) into Eq. (7), the analytical expression of the electrical potential in the aqueous solution can be obtained [52]:

$$
\phi=-E_{\infty} r \cos \theta-\frac{1}{2} E_{\infty} \frac{a^{3}}{r^{2}} \cos \theta
$$

The electric potential on the oil droplet $\phi(\theta)$ can be given by:

$$
\phi(\theta)=-\frac{3}{2} E_{\infty} a \cos \theta
$$

\subsection{Local zeta potential on oil droplet}

As mentioned above, under the influence of the electric field, the mobile surface charges on the oil droplet get redistributed; hence, the counter ions inside EDL. The redistributed ions generate local electric field on the oil droplet which repels the externally applied electric field. At equilibrium state, all field lines of the externally applied electric field get expelled. Therefore, the local electric field $E_{i}$ on the oil droplet has the same magnitude and is in the opposite direction to that of externally applied electrical field $E_{e}$, as indicated by the following equation:

$$
E_{i}=-E_{e}
$$

or,

$$
\nabla \zeta(\theta)=-\nabla \phi(\theta)
$$

where $\zeta(\theta)$ is the local zeta potential, $\phi(\theta)$ is the electric potential of the externally applied electrical field. In the spherical coordinate system, the expression of $\phi(\theta)$ is given by Eq. (12). Integrating Eq. (14), the local zeta potential can be obtained as:

$$
\zeta(\theta)=-\phi(\theta)+\phi_{c}
$$

where $\phi_{c}$ is an integration constant. 
As described above, for a negatively charged oil droplet, under externally applied electrical field, the surface charges move and accumulate to the side of the oil droplet facing the anode of the electrical field, and the surface charge density on the other side decreases, especially at the pole facing the cathode of the electric field. As the surface charges move away, the zeta potential on the side facing the cathode decreases, and the chemical equilibrium at the oil-water interface is broken. In order to reach equilibrium again, the oil-water interface on this side starts recharging by adsorbing the negative hydroxide ions from the bulk water. The recharging process will continue until reaching the equilibrium state. It should be realized that the initial zeta potential is the equilibrium zeta potential determined by the given bulk ionic concentration and the given $\mathrm{pH}$ value. This zeta potential must be reached at the point where the minimum zeta potential stays; otherwise, the recharging process will not stop. Therefore, at equilibrium state, the minimum local zeta potential on the oil droplet needs to be equal to the initial zeta potential of the oil droplet before applying electric field. Under externally applied electric field, the minimum zeta potential stays at the pole facing the cathode $(\theta=0)$. Hence, the following equation can be obtained:

$$
\zeta(\theta=0)=\zeta_{0}
$$

where $\zeta_{0}$ is the initial zeta potential of the oil droplet before the external electric field is applied. With this boundary condition, Eq. (15) can be solved. The integration constant $\phi_{c}$ can be determined by:

$$
\phi_{c}=\zeta_{0}+\phi(0)=\zeta_{0}-\frac{3}{2} E_{\infty} a
$$

Substituting Eq. (17) into Eq. (15), the redistributed local zeta potential along the surface of the oil droplet can be written as:

$$
\zeta(\theta)=\frac{3}{2} E_{\infty} a(\cos \theta-1)+\zeta_{0}
$$

\subsection{Local surface charge density on oil droplet}

As shown in Figure 3, when a flat surface is immersed in an aqueous solution, it carries negative charges and the electric double layer forms in vicinity of it. A coordinate system is built on the surface. The origin point of the coordinate system locates on the surface, and the $\mathrm{x}$-axis is perpendicular to the flat surface pointing to the water phase. At stable state, the surface charge density on the surface $\sigma_{0}$ should be equal to the net charges in the electrolyte, which yields the following equation: 


$$
\sigma_{0}=-\int_{0}^{\infty} \rho_{e} d x
$$

where $\rho_{e}$ is the net charge density in the electrolyte.

The relationship between the electrical potential of the electric double layer field $\psi$ and the net charge density $\rho_{e}$ is given by the well-known Poisson's equation:

$$
\frac{d^{2} \psi}{d x^{2}}=-\frac{\rho_{e}}{\varepsilon_{0} \varepsilon_{m}}
$$

Substituting Eq. (20) into Eq. (19), the expression of $\sigma_{0}$ in terms of $\psi$ can be obtained:

$$
\sigma_{0}=-\varepsilon_{0} \varepsilon_{m} \int_{0}^{\infty} \frac{d^{2} \psi}{d x^{2}} d x
$$

As $d \psi / d x \rightarrow 0$ at $x \rightarrow \infty$, the above equation can be integrated to:

$$
\sigma_{0}=-\varepsilon_{0} \varepsilon_{m}\left(\frac{d \psi}{d x}\right)_{x=0}
$$

The electrical potential of the EDL field near the flat surface $\psi$ can be calculated by the onedimensional Poisson-Boltzmann equation:

$$
\frac{d^{2} \psi}{d x^{2}}=\frac{2 z e n_{\infty}}{\varepsilon_{0} \varepsilon_{m}} \sinh \left(\frac{z e \psi}{K_{b} T}\right)
$$

Intergrating this equation yields:

$$
\frac{d \psi}{d x}=-\frac{4 n_{\infty} z e}{\varepsilon_{0} \varepsilon_{m} \kappa} \sinh \left(\frac{z e \psi}{2 K_{b} T}\right)
$$

where $\kappa$ is the Debye-Huckel parameter. Substituting Eq. (24) into Eq. (22), the equation of the surface charge density can be obtained:

$$
\sigma_{0}=\frac{4 n_{\infty} z e}{\kappa}\left(\sinh \left(\frac{z e \psi}{2 K_{b} T}\right)\right)_{x=0}
$$

On the flat surface $(x=0), \psi \approx \zeta_{s}$; therefore, the surface charge density as functions of the zeta potential can be obtained as [1]: 


$$
\sigma_{0}=\frac{4 n_{\infty} z e}{\kappa} \sinh \left(\frac{z e \psi \zeta_{s}}{2 K_{b} T}\right)
$$

For the oil droplet, we consider the droplet size is much larger than the thickness of electric double layer, and hence the curved droplet surface may be approximated as a flat surface. Therefore, the local surface charge density $\sigma(\theta)$ in terms of the local zeta potential $\zeta(\theta)$ can be obtained:

$$
\sigma(\theta)=\frac{4 n_{\infty} z e}{\kappa} \sinh \left(\frac{z e \zeta(\theta)}{2 K_{b} T}\right)
$$

Using the Debye-Huckle linear approximation, the above equation can be reduced to:

$$
\sigma(\theta)=\frac{2 n_{\infty}(z e)^{2}}{\kappa K_{b} T} \zeta(\theta)
$$

As shown in this equation, for a given oil-water system $\left(\varepsilon_{m}, n_{\infty}, z\right.$ and $T$ are constants), the local surface charge density is linear proportional to the local zeta potential. Substituting Eq. (18) into Eq. (28), the local surface charge density along the surface of the oil droplet under externally applied electric field can be got as:

$$
\sigma(\theta)=\frac{2 n_{\infty}(z e)^{2}}{\kappa K_{b} T}\left[\frac{3}{2} E_{\infty} a(\cos \theta-1)+\zeta_{0}\right]
$$

As indicated in Eq. (18), the local zeta potential at the point $\theta=0$ is a constant which is equal to $\zeta_{0}$. The redistributed local zeta potential along the surface of the oil droplet is related to the initial zeta potential, and depends on the dielectric permittivity of the drop and the surrounding medium, the radius of the oil droplet, and the applied electric field. While keeping other parameters fixed, the increase of the droplet radius $(a)$ and the applied electric field $\left(E_{\infty}\right)$ will increase the redistributed local zeta potential. By comparing Eq. (18) with Eq. (29), one can see that the variation of the local surface charge density is proportional to the local zeta potential. The surface charge redistribution model shown in this paper was derived under the assumptions of thin electric double layer $(a \gg 1 / \kappa)$ and weak strength of externally applied electrical field. Under these conditions, the electrophoretic motion of the negatively charged oil droplet is so slow that it does not affect the ion distribution inside the thin electric double layer around the oil 
droplet. Therefore, the distributions of surface charges and ions insider electric double layer are considered to be unchanged with the weak electrophoretic motion of the oil droplet.

\section{Results and discussion}

An analytical model of the surface charge redistribution on a spherical oil droplet in water under electric field was derived above. Examination of these equations reveals that the local zeta potential along the surface of the oil droplet is determined by following parameters: $\varepsilon_{m}, a, \zeta_{0}$ and $E_{\infty}$. Here $\varepsilon_{m}$ is the relative dielectric permittivity of the surrounding aqueous solution, which is a constant for a given oil-water system. Therefore, the effects of the initial zeta potential $\zeta_{0}$, radius $a$ and the applied electrical field strength $E_{\infty}$ on the redistribution of the mobile surface charges will be analyzed. In the following discussions, the relative dielectric constant of water $\varepsilon_{m}$ is taken as 80 . The local zeta potential along the surface of oil droplet can be calculated by using the equation of the developed model, i.e., Eq. (18), with a set of values of the initial zeta potential, the droplet radius, and the externally applied electric field.

\subsection{Effect of the initial zeta potential}

Figure 4 shows the distributions of local zeta potential along the surface of an oil droplet of $10 \mu \mathrm{m}$ in radius for two different initial zeta potentials under $E_{\infty}=20 \mathrm{~V} / \mathrm{cm}$. The solid line indicates the zeta potential redistribution with an initial zeta potential of $\zeta_{0}=-30 \mathrm{mV}$, and the dash line shows the redistributed zeta potential along the oil droplet with the initial zeta potential of $-60 \mathrm{mV}$. As it is seen in Figure 4, the redistributed local zeta potential has its maximum value at the pole facing the applied electrical field at $\theta=180^{\circ}$; the local zeta potential has its minimum value at the other pole at $\theta=0^{\circ}$, which equals to the initial zeta potential $\zeta_{0}$. The comparison of the two curves indicates that the redistributed local zeta potential increases with the increase of the initial zeta potential. This can be easily understood. Because a higher initial zeta potential indicates a higher initial surface charge density and hence a higher total number of mobile surface charges on the droplet, consequently, more negative surface charges will be attracted and accumulated near the pole facing the positive electrode, resulting a higher local zeta potential at this pole at $\theta=180^{\circ}$ to balance the externally applied electrical field. While the negative surface charges are moving away from the side of oil droplet facing the negative 
electrode, new surface charges are created on this side of the surface until the zeta potential at the pole $\theta=0^{\circ}$ equals to the initial zeta potential of the oil droplet.

\subsection{Effect of the droplet radius}

Droplet size is another factor that affects the surface charge redistribution. The externally applied electric field along the surface of an oil droplet $E_{e}$ can be obtained by differentiating Eq. (12):

$$
E_{e}=\nabla \phi(\theta)=\frac{3}{2} E_{\infty} a \sin \theta
$$

Clearly, $E_{e}$ as the driving force for the redistribution of the mobile surface charges is dependent on the droplet size. Under a given electrical field $\left(E_{\infty}=\right.$ constant $)$, smaller of the droplet size, smaller the driving force. Therefore, for oil droplets with fixed initial zeta potential under the same externally applied electric field, except at the pole facing the cathode, the local zeta potential of the oil droplet decreases with the decrease of the oil droplet size.

The zeta potential distribution around oil droplets of different radius is shown in Figure 5. The zeta potential distributions along the surface oil droplets of $1 \mu \mathrm{m}, 5 \mu \mathrm{m}$ and $10 \mu \mathrm{m}$ in radius under externally applied electric field $E_{\infty}=20 \mathrm{~V} / \mathrm{cm}$ are indicated by the solid line, dash line and dotdashed line, respectively. Figure 5 clearly shows two points: (1) For fixed initial zeta potential of oil droplets, the local zeta potential at the pole of the droplet facing the negative electrode $\left(\theta=0^{\circ}\right)$ is a constant which equals to the initial zeta potential and doesn't vary with the radius of the oil droplet; (2) The value of the local zeta potential on the rest surface of the droplet increases with the droplet radius. As shown in Figure 5, the maximum value of the redistributed local zeta potential is $-56 \mathrm{mV}$ for the oil droplet of $1 \mu \mathrm{m}$ in radius with an initial zeta potential of $-50 \mathrm{mV}$ under electric field $E_{\infty}=20 \mathrm{~V} / \mathrm{cm}$. For the oil droplets with a radius of $5 \mu \mathrm{m}$ and $10 \mu \mathrm{m}$, respectively, with the same initial zeta potential and under the same electric field, the maximum zeta potential increases to $-80 \mathrm{mV}$ and $-110 \mathrm{mV}$, respectively.

\subsection{Effect of the applied electric field}

For a droplet with a fixed size and a fixed initial zeta potential, the redistribution of the mobile surface charges and the zeta potential changes with the strength of applied electric field. The effect of the applied electric field can be understood as follows. When the applied electric field changes, the electrostatic driven force acting on the mobile surface charges of the oil droplet will 
change, hence affect the distribution and concentration of the surface charges, as well as the zeta potential redistribution along the surface of oil droplet. For example, when the electric field increases, larger local electric field generated by the accumulated surface charges is needed to balance the externally applied electric field, which will cause the concentration of the surface charges increase.

The zeta potential distributions around the same oil droplet under three different applied electric fields are shown in Figure 6. The solid, dash and dot-dashed curves represent the distribution of zeta potential when the applied electric field are $10 \mathrm{~V} / \mathrm{cm}, 20 \mathrm{~V} / \mathrm{cm}$ and $50 \mathrm{~V} / \mathrm{cm}$, respectively. For $E_{\infty}=10 \mathrm{~V} / \mathrm{cm}$, the local zeta potential increases gradually from the pole $\theta=0^{\circ}(\zeta(\theta=$ $\left.\left.0^{\circ}\right)=-50 \mathrm{mV}\right)$ to the other pole $\theta=180^{\circ}\left(\zeta\left(\theta=180^{\circ}\right)=-65 \mathrm{mV}\right)$. If increasing $E_{\infty}$ to $50 \mathrm{~V} / \mathrm{cm}$ and keeping the other parameters unchanged, the local zeta potential at $\theta=0^{\circ}$ remains the same and the maximum value of the local zeta potential at $\theta=180^{\circ}$ can reach $-125 \mathrm{mV}$.

\section{Experimental validation}

Considering the limitations of the existing methods of visualizing the surface charges as reviewed in the introduction, nanoparticles were used in this paper to analogize the mobile charged molecules at oil-water interface. Similar to the charged molecules at the oil-water interface, the charged nanoparticles will move and accumulate to one pole of the oil droplet under externally applied electric field, and a "blank surface area" will show up on the other side. By visualizing the motion and accumulation of nanoparticles on the surface of oil drops under externally applied electric field, the predication of the redistribution of the mobile surface charges by the model developed in this paper may be validated.

\subsection{Model modification}

Although the nanoparticles can be used to simulate the motion and redistribution of the charged molecules at oil-water interface under electric field, the nanoparticles cannot be regenerated on the "blank surface area" after they have moved and accumulated in one pole of the droplet. This is because the total number of the nanoparticles attached to an oil droplet surface is a constant, which is different from the number of charged molecules on the oil droplet surface. When the mobile charged molecules migrate away from one surface area, attracted by the applied electric field, new charged molecules will be formed in that area to maintain a local chemical equilibrium 
of the oil-water interface for the given conditions (e.g., pH). To address this difference, the redistribution of the charged nanoparticles will be estimated by modifying the above-described mobile charge redistribution model with a different boundary condition. As positively charged aluminum oxide nanoparticles will be used to analogize the charged molecules at the oil-water interface, we will assume the oil-water interface carries positively charges which will accumulate to the side of oil droplet facing cathode, as shown in Figure 7. Furthermore, in order to consider the fact that the nanoparticles cannot be regenerated on the "blank surface area", we will assume that the total amount of surface charges remains constant before and after the external electric field is applied. Therefore, Eq. (15) will be solved with the boundary condition that the total number of surface charges on the oil droplet, $Q$, must equal to the initial number of surface charges, $Q_{0}$ :

$$
Q=Q_{0}
$$

For an oil droplet immersed in an aqueous solution with zeta potential $\zeta_{0}$ initially (i.e., before applying the electrical field), the total amount of surface charges uniformly distributed on the droplet surface can be calculated by using the following equation [1,3]:

$$
Q_{0}=4 \pi \varepsilon_{m} \varepsilon_{o} a(1+\kappa a) \zeta_{0}
$$

For the purpose of modeling the redistribution of the nanoparticles on the droplet surface, we assume that, after applying the electrical field (from left to right), all the positive mobile surface charges are moved towards the right hand side of the droplet, and the surface on the left-hand side of the droplet is free of surface charges (i.e., to simulate the condition that the nanoparticles cannot be regenerated there), as shown in Figure 7 . Let us assume $\theta 1$ is the boundary of the surface area with the redistributed surface charges. At this boundary, the local zeta potential is zero, i.e., $\zeta(\theta 1)=0$. Substituting this boundary condition into Eq. (15) yields:

$$
\phi_{c}=\phi(\theta 1)=-\frac{3}{2} E_{\infty} a \cos \theta 1
$$

Therefore, the expression of the local zeta potential $\zeta(\theta)$ is given by:

$$
\zeta(\theta)=\frac{3}{2} E_{\infty} a \cos \theta-\frac{3}{2} E_{\infty} a \cos \theta 1
$$


The relationship between the local surface charge density $\sigma(\theta)$ and the local zeta potential $\zeta(\theta)$ is given by Eq. (28). Therefore, the redistributed local surface charge density can be got by substituting Eq. (34) into Eq. (28):

$$
\sigma(\theta)=\frac{2 n_{\infty}(z e)^{2}}{\kappa K_{b} T}\left(\frac{3}{2} E_{\infty} a \cos \theta-\frac{3}{2} E_{\infty} a \cos \theta 1\right)
$$

The total amount of surface charges of the droplet $Q$ can be calculated:

$$
Q=\int \sigma(\theta) d A=2 \pi a^{2} \int_{0}^{\theta 1} \sigma(\theta) \sin \theta d \theta
$$

here, $A$ is the surface area where the surface charges accumulate.

Substituting Eq. (35) into Eq. (36) yields the expression of $Q$ in terms of $\theta$ as the following:

$$
Q=6 \pi a^{2} \frac{n_{\infty}(z e)^{2}}{\kappa K_{b} T} E_{\infty} a \int_{0}^{\theta 1}(\cos \theta-\cos \theta 1) \sin \theta d \theta
$$

As the total amount of surface charges does not change before and after the electric field is applied (i.e., Eq. (31)), Eq. (32) is equal to Eq. (37).With this relationship, $\theta 1$ can be determined from the equation below:

$$
\cos \theta 1=1-\sqrt{\frac{4}{3} \cdot \frac{\varepsilon_{o} \varepsilon_{m}(1+\kappa a) \kappa K_{b} T}{a^{2} n_{\infty}(z e)^{2} E_{\infty}} \zeta_{0}}
$$

For thin EDL, $\kappa a \gg 1$, the equation can be reduced to:

$$
\cos \theta 1=1-\sqrt{\frac{4}{3} \cdot \frac{\varepsilon_{0} \varepsilon_{m} \kappa^{2} K_{b} T}{a n_{\infty}(z e)^{2} E_{\infty}} \zeta_{0}}
$$

As indicated by Eq. (39), the boundary of the charge accumulation area $\theta 1$ is a function of the initial zeta potential $\zeta_{0}$, the dielectric permittivity of the surrounding medium, the radius of the oil droplet, and the applied electric field. While keeping other parameters fixed, the increase of the applied electric field $\left(E_{\infty}\right)$ will reduce the charge accumulation surface area $(\theta 1)$. The variation of the surface area covered with the redistributed surface charges in response to the externally applied electric is shown in Figure 8. It is clearly shown that the increase of the electric field leads to a smaller area of the droplet surface with the redistributed charges. 


\subsection{Nanoparticles and experimental procedures}

In the experiments, $\mathrm{Al}_{2} \mathrm{O}_{3}$-passivated aluminum nanoparticles (Skyspring Nanomaterials, Inc., Houston, TX, USA) with an average diameter of $18 \mathrm{~nm}$ were used. These nanoparticles carry positive charges when they are in contact with deionized water, with a zeta potential of approximately $+60 \mathrm{mV}[2,53,54]$. The reason for choosing the positive charged nanoparticles is because they can be easily deposited on the negatively charged oil-water interface. In contrary, negatively charged nanoparticles are difficult to do so.

In the experiments, $2 \mathrm{mg}$ nanoparticles were added into $1 \mathrm{~mL}$ deionized water, and then placed in an ultrasonic cleaner for 8 minutes to disperse the particles The experimental procedures include the following:

a) To increase the wettability of water on polystyrene surface, a plasma cleaner (HARRIC PLASMA, Ithaca, NY, USA) is used to do surface treatment to a polystyrene petri dish for $3 \mathrm{mins}$;

b) Deposit $2.5 \mu \mathrm{L}$ oil (Nikon Type A immersion oil) by a digital micropipette on the bottom surface of the plasma-cleaned polystyrene petri dish. A sessile drop of oil forms with a radius approximately $0.6 \mathrm{~mm}$.

c) Pour deionized water into the petri dish until the water level is around $5 \mathrm{~mm}$ above the top surface of the oil drop. As the oil droplet has already attached on the surface of the petri dish, it will not float.

d) Deposit $1.5 \mu \mathrm{L}$ of nanoparticle suspension (with a concentration of $2 \mathrm{mg} / \mathrm{mL}$ ) by a digital micropipette on the top of the oil droplet. Wait $5 \mathrm{~min}$ for the particles to settle on the interface.

e) Insert electrodes into the water phase and apply electric field $E=10 \mathrm{~V} / \mathrm{cm}$ from left to right. Use a microscope (Nikon Ti-E) imaging system to observe and record the redistribution of the nanoparticles at the oil-water interface.

It should be note that the method for visualizing the surface charges is limited. Therefore, the nanoparticles are used to analogize the mobile charged molecules at oil-water interface here. The redistribution of the mobile surface charges can be validated by visualizing the motion and accumulation of nanoparticles on the surface of oil droplet under externally applied electrical field. 
From a macroscopic view, the aluminum oxide nanoparticles on the oil droplet can be analogous to positive charges on the oil droplet, in terms of their response to the applied electric field. As the nanoparticles cannot be regenerated on the oil-water interface after they move away and accumulate to one side of the oil droplet under externally applied electrical field, the modified model shown above considers that the total number of the nanoparticles remains fixed. With this condition, the analytical equation of the boundary of the charge accumulation area is derived. Therefore, the modified model shown in the experimental part is suitable for evaluating the nanoparticle accumulation surface area under externally applied electrical field.

However, from a microscopic view, the size of the nanoparticles cannot be neglected. Generally, the effective size of a molecule ranges from $0.1 \mathrm{~nm}$ to a few nanometers. The average diameter of the $\mathrm{Al}_{2} \mathrm{O}_{3}$-passivated aluminum nanoparticles used in the experiment is $18 \mathrm{~nm}$, which is 10 to 100 times larger than the charged molecules. In the experiments, positively charge nanoparticles were used to cover the negatively charged oil droplet. Figure 9(a) shows the schematic of the distribution of the positively charged nanoparticles at the oil-water interface before applying electric field. As shown in this figure, the nanoparticles and the charged molecules stay on different layers, and the major part of the positively charged nanoparticles stays in the water phase. Under externally applied electrical field, the positively charged nanoparticles are driven to move and accumulate to one side of the oil droplet facing the cathode of the electric field, as shown in Figure 9(b). When the nanoparticles get packed close to each other, the electric field lines around the nanoparticles are also affected. As shown in Figure 9(b), the external electric field lines detour around the nanoparticles, no line can enter into the small gap between the two nanoparticles and act on the oil-water interface. Meanwhile, the accumulated positively charged nanoparticles act as an electric shield screen, a local electrical field is built which expels the external electric field lines. At equilibrium state, all of the electric field lines are repelled and the externally applied electrical field on that side is balanced by the local electric field generated by the redistributed nanoparticles on that side of the oil droplet. Therefore, in the nanoparticle accumulation region, the packed nanoparticle layer performs as the shielding layer which prevents the external electric field interacting with the surface charges of the oil-water interface. Without the effect of the electric field, the mobile surface charges in the nanoparticle accumulation region of the oil droplet are uniformly distributed, and the effect of the mobile surface charges in this region can be neglected. 


\subsection{Experiment results}

The typical redistribution of the positively charged aluminum nanoparticles in response to the externally applied electric field are shown by the sequence of images in Figure 10. Before the electric field was applied at $t=0$, the nanoparticles at the oil-water interface are uniformly distributed and motionless, as shown in Figure 10(a). After the electric field was turned on, the positively charged nanoparticles at the interface were observed to move in the same direction as the applied electric field, i.e., from left to right (Figures 10(b) (e)). As time went by, more and more nanoparticles moved to the right side and accumulated at the right hemisphere of the droplet until reaching the final state, as shown in Figure 10(f). At the final state, all of the nanoparticles accumulated to the right hemisphere and a large "blank area" without the presence of nanoparticles was left in the left hemisphere of the droplet. It should be pointed out that the slow movement of the nanoparticles is because the size of the nanoparticles is tens of times larger than that of the charged molecules such as $\mathrm{Na}^{+}$. However, such a redistribution of the charged nanoparticles at the oil-water interface in response to the external electrical field is analogous to that of the mobile surface charge at a liquid-fluid interface.

Based on the spherical shape assumption and the measured drop dimensions, the boundary of the nanoparticle accumulation area in the final state (Figure 10(f)) can be evaluated to be approximately $\theta 1=65^{\circ}$. It must be pointed out that the theoretical models developed in this paper are valid only for mobile surface charges of common molecular sizes, and the nanoparticles are many times larger than molecules. Theoretically, the models are not applicable to predict the behavior of the mobile nanoparticles on the oil-water interface. With this understanding, however, it is desirable to see if the models developed in this paper would be able to provide qualitative estimation of the nanoparticle redistribution. If one uses the aluminum nanoparticles' zeta potential $60 \mathrm{mV}$ as the initial zeta potential, the corresponding $\theta 1$ for the specific condition of droplet radius of $a=0.6 \mathrm{~mm}$ and $E_{\infty}=10 \mathrm{~V} / \mathrm{cm}$ can be calculated by using Eq. (39); the $\theta 1$ value is $61.1^{\circ}$. The comparison of the model predicted result $\left(61.1^{\circ}\right)$ with the experimental result $\left(65^{\circ}\right)$ indicates a good agreement. 


\section{Conclusion}

This paper presents a fundamental investigation on the redistribution of mobile surface charges of an oil droplet in water under externally applied electric field. A model was derived based on the balance between the local electric field $E_{i}$ generated by the redistributed surface charges and the local externally applied electrical field $E_{e}$. With the model, the effects of the initial zeta potential, the radius of oil droplet as well as the applied electric field on the redistribution of the mobile surface charges were investigated analytically. It was found that the redistribution of surface charges strongly depends on these three parameters. Generally, increase in oil droplet radius and electric field makes the redistributed local zeta potential increase. A larger initial zeta potential results in larger redistributed local zeta potential along the spherical oil droplet under the same applied electric field.

The redistribution of mobile surface charges was validated experimentally by using charged nanoparticles to analogize the charged molecules at the oil-water interface. The experimental result indicates that the charged nanoparticles will migrate and accumulate to one pole of the oil droplet under externally applied electric field, which is similar to our theoretical analysis. However, the difference between the charged nanoparticles and the charged molecules at oilwater interfaces is that the nanoparticles cannot be regenerated after migrating away. To estimate the accumulation of charged nanoparticles, the model was modified by considering the boundary condition that the total amount of mobile charged nanoparticles remained constant before and after the electric field was applied and the expression of the surface area with redistributed charged particles was derived. The surface area with the accumulated nanoparticles was measured experimentally and is in good agreement with the predication of the model developed in this paper.

\section{Acknowledgements}

The authors wish to thank the financial support of the Natural Sciences and Engineering Research Council (NSERC) of Canada through a research grant to D. Li. 


\section{References}

[1] Li D. Electrokinetics in Microfluidics. London: Elsevier Ltd.; 2004.

[2] Hunter RJ. Zeta Potential in Colloid Science: Principles and Applications. New York: Academic Press; 1981.

[3] Arulanandam S, Li D. Liquid transport in rectangular microchannels by electroosmotic pumping. Colloids Surfaces A Physicochem Eng Asp 2000;161:89-102. doi:10.1016/S0927-7757(99)00328-3.

[4] Hsu JP, Kao CY, Tseng S, Chen CJ. Electrokinetic flow through an elliptical microchannel: effects of aspect ratio and electrical boundary conditions. J Colloid Interface Sci 2002;248:176-84. doi:10.1006/jcis.2001.8200.

[5] Kang Y, Yang C, Huang X. Electroosmotic flow in a capillary annulus with high zeta potentials. J Colloid Interface Sci 2002;253:285-94. doi:10.1006/jcis.2002.8453.

[6] Tsao HK. Electroosmotic Flow through an Annulus. J Colloid Interface Sci 2000;225:247-50. doi:10.1006/jcis.1999.6696.

[7] Baygents JC, Saville DA. Electrophoresis of small particles and fluid globules in weak electrolytes. J Colloid Interface Sci 1991;146:9-37. doi:10.1016/0021-9797(91)90003-Q.

[8] Ye C, Li D. Electrophoretic motion of a sphere in a microchannel under the gravitational field. J Colloid Interface Sci 2002;251:331-8. doi:10.1006/jcis.2002.8438.

[9] Ennis J, Zhang H, Stevens G, Perera J, Scales P, Carnie S. Mobility of protein through a porous membrane. J Memb Sci 1996;119:47-58. doi:10.1016/0376-7388(96)00112-3.

[10] Creux P, Lachaise J, Graciaa A, Beattie JK, Djerdjev AM. Strong Specific Hydroxide Ion Binding at the Pristine Oil / Water and Air / Water Interfaces. J Phys Chem B 2009;113:14146-50. doi:10.1021/jp906978v.

[11] Marinova K, Alargova R, Denkov N. Charging of oil-water interfaces due to spontaneous adsorption of hydroxyl ions. Langmuir 1996;12:2045-51. doi:10.1021/la950928i.

[12] Fang H, Wu W, Sang Y, Chen S, Zhu X, Zhang L, et al. Evidence of the Adsorption of Hydroxide Ion at Hexadecane/Water Interface from Second Harmonic Generation Study. RSC Adv 2015;5:23578-85. doi:10.1039/C4RA15401K.

[13] Ma A, Xu J, Xu H. Impact of spontaneously adsorbed hydroxide ions on emulsification via solvent shifting. J Phys Chem C 2014;118:23175-80. doi:10.1021/jp5076109. 
[14] Stachurski J, MichaLek M. The Effect of the zeta Potential on the Stability of a Non-Polar Oil-in-Water Emulsion. J Colloid Interface Sci 1996;184:433-6. doi:10.1006/jcis.1996.0637.

[15] Beattie JK, Djerdjev AM. The pristine oil/water interface: Surfactant-free hydroxidecharged emulsions. Angew Chemie - Int Ed 2004;43:3568-71. doi:10.1002/anie.200453916.

[16] Roger K, Cabane B. Why Are Hydrophobic/Water Interfaces Negatively Charged? Angew Chemie Int Ed 2012;51:5625-8. doi:10.1002/anie.201108228.

[17] Roger K, Cabane B. Uncontaminated Hydrophobic/Water Interfaces Are Uncharged: A Reply. Angew Chemie 2012;124:13117-9. doi:10.1002/ange.201207114.

[18] Gao Y, Wong TN, Yang C, Ooi KT. Transient two-liquid electroosmotic flow with electric charges at the interface. Colloids Surfaces A Physicochem Eng Asp 2005;266:117-28. doi:10.1016/j.colsurfa.2005.05.068.

[19] Lee JSH, Barbulovic-Nad I, Wu Z, Xuan X, Li D. Electrokinetic flow in a free surfaceguided microchannel. J Appl Phys 2006;99:54905. doi:10.1063/1.2177428.

[20] Brask A, Goranovic G, Bruus H. Electroosmotic pumping of nonconducting liquids by viscous drag from a secondary conducting liquid. Proc. NanoTech, vol. 1, San Francisco, USA: 2003, p. 190-3.

[21] Gao Y, Wong TN, Chai JC, Yang C, Ooi KT. Numerical simulation of two-fluid electroosmotic flow in microchannels. Int J Heat Mass Transf 2005;48:5103-11. doi:10.1016/j.ijheatmasstransfer.2005.08.005.

[22] Lee JSH, Li D. Electroosmotic flow at a liquid-air interface. Microfluid Nanofluid 2006;2:361-5. doi:10.1007/s10404-006-0084-9.

[23] Movahed S, Khani S, Wen JZ, Li D. Electroosmotic flow in a water column surrounded by an immiscible liquid. J Colloid Interface Sci 2012;372:207-11. doi:10.1016/j.jcis.2012.01.044.

[24] Gao Y, Wang C, Wong TN, Yang C, Nguyen N, Ooi KT. Electro-osmotic control of the interface position of two-liquid flow through a microchannel. $\mathrm{J}$ Micromechanics Microengineering 2007;17:358-66. doi:10.1088/0960-1317/17/2/023.

[25] Li H, Teck NW, Nguyen NT. Time-dependent model of mixed electroosmotic/pressuredriven three immiscible fluids in a rectangular microchannel. Int J Heat Mass Transf 2010;53:772-85. doi:10.1016/j.ijheatmasstransfer.2009.10.011.

[26] Qian S, Joo SW, Jiang Y, Cheney MA. Free-surface problems in electrokinetic micro- and nanofluidics. Mech Res Commun 2009;36:82-91. doi:10.1016/j.mechrescom.2008.06.014. 
[27] Choi WS, Sharma A, Qian S, Lim G, Joo SW. Is free surface free in micro-scale electrokinetic flows? J Colloid Interface Sci 2010;347:153-5. doi:10.1016/j.jcis.2010.03.049.

[28] Ray B, Reddy PDS, Bandyopadhyay D, Joo SW, Sharma A, Qian S, et al. Instabilities in free-surface electroosmotic flows. Theor Comput Fluid Dyn 2012;26:311-8. doi:10.1007/s00162-011-0234-x.

[29] Beloqui Redondo A, Jordan I, Ziazadeh I, Kleibert A, Giorgi JB, Wörner HJ, et al. Nanoparticle-Induced Charge Redistribution of the Air-Water Interface. J Phys Chem C 2015;119:2661-8. doi:10.1021/jp511915b.

[30] Xu H, Kirkwood J, Lask M, Fuller G. Charge interaction between particle-laden fluid interfaces. Langmuir 2010;26:3160-4. doi:10.1021/la903099a.

[31] Ichikawa T, Nakajima Y. Rapid demulsification of dense oil-in-water emulsion by low external electric field. I. Experimental evidence. Colloids Surfaces A Physicochem Eng Asp 2004;242:21-6. doi:10.1016/j.colsurfa.2004.04.042.

[32] Ichikawa T, Nakajima Y. Rapid demulsification of dense oil-in-water emulsion by low external electric field. II. Theory. Colloids Surfaces A Physicochem Eng Asp 2004;242:27-37. doi:10.1016/j.colsurfa.2004.04.042.

[33] Ichikawa T, Dohda T, Nakajima Y. Stability of oil-in-water emulsion with mobile surface charge. Colloids Surfaces A Physicochem Eng Asp 2006;279:128-41. doi:10.1016/j.colsurfa.2005.12.050.

[34] Ichikawa T. Electrical demulsification of oil-in-water emulsion. Colloids Surfaces A Physicochem Eng Asp 2007;302:581-6. doi:10.1016/j.colsurfa.2007.03.036.

[35] Camp JP, Capitano AT. Size-dependent mobile surface charge model of cell electrophoresis. Biophys Chem 2005;113:115-22. doi:10.1016/j.bpc.2004.07.034.

[36] Jaffe LF. Electrophoresis along cell membranes. Nature 1977;265:600-2. doi:10.1038/265600a0.

[37] Poo M, Lam JW, Orida N, Chao AW. Electrophoresis and Diffusion in the plane of the cell membrane. Biophys J 1979;26:1-22.

[38] Poo M. In situ electrophoresis of membrane components. Annu Rev Biophys Bioeng 1981;10:245-76. doi:10.1146/annurev.bb.10.060181.001333.

[39] Gross D. Electromobile surface charge alters membrane potential changes induced by applied electric fields. Biophys J 1988;54:879-84. doi:10.1016/S0006-3495(88)83024-8. 
[40] Kazoe Y, Sato Y. Effect of ion motion on zeta-potential distribution at microchannel wall obtained from nanoscale laser-induced fluorescence. Anal Chem 2007;79:6727-33. doi:10.1021/ac070547x.

[41] Kazoe Y, Miyakawa S, Miki N, Sato Y. Fluorescence imaging technique of surface electrostatic potential using evanescent wave illumination. Appl Phys Lett 2009;95:95-8. doi:10.1063/1.3266842.

[42] Shieh IC, Zasadzinski JA. Visualizing monolayers with a water-soluble fluorophore to quantify adsorption, desorption, and the double layer. Proc Natl Acad Sci 2015;112:E82635. doi:10.1073/pnas.1419033112.

[43] Webb RH. Confocal optical microscopy. Reports Prog Phys 1996;59:427-71. doi:10.1088/0034-4885/59/3/003.

[44] Luo G, Malkova S, Yoon J, Schultz DG, Lin B, Meron M, et al. Ion distributions near a liquid-liquid interface. Science 2006;311:216-8. doi:10.1126/science.1120392.

[45] Lee KYC, Majewski J, Kuhl TL, Howes PB, Kjaer K, Lipp MM, et al. Synchrotron X-ray study of lung surfactant-specific protein SP-B in lipid monolayers. Biophys J 2001;81:572-85. doi:10.1016/S0006-3495(01)75724-4.

[46] Laanait N, Mihaylov M, Hou B, Yu H, Vanýsek P, Meron M, et al. Tuning ion correlations at an electrified soft interface. Proc Natl Acad Sci 2012;109:20326-31. doi:10.1073/pnas.1214204109.

[47] Pereira CM, Martins A, Rocha M, Silva CJ, Silva F. Differential capacitance of liquid/liquid interfaces: effect of electrolytes present in each phase. J Chem Soc Faraday Trans 1994;90:143-8. doi:10.1039/ft9949000143.

[48] Vogel V, Möbius D. Local Surface Potentials and Electric Dipole Moments of Lipid Monolayers : Contributions of the Water / Lipid and the Lipid / Air Interfaces. J Colloid Interface Sci 1988;126:408-20. doi:10.1016/0021-9797(88)90140-3.

[49] Samec Z, Marecek V, Homolka D. The double layer at the interface between two immiscible electrolyte solutions: Part II. Structure of the water/nitrobenzene interface in the presence of 1:1 and 2:2 electrolytes. J Electroanal Chem 1985;187:31-51.

[50] Diaz RE. Electrostatics. Electr Eng Handb 2005;1:499-512. doi:10.1016/B978012170960-0/50038-4.

[51] Wu Z, Li D. Mixing and flow regulating by induced-charge electrokinetic flow in a microchannel with a pair of conducting triangle hurdles. Microfluid Nanofluid 2008;5:6576. doi:10.1007/s10404-007-0227-7. 
[52] Zhang F, Li D. Induced-charge electroosmotic flow around dielectric particles in uniform electric field. J Colloid Interface Sci 2013;410:102-10. doi:10.1016/j.jcis.2013.08.017.

[53] Timofeeva E V., Gavrilov AN, McCloskey JM, Tolmachev Y V., Sprunt S, Lopatina LM, et al. Thermal conductivity and particle agglomeration in alumina nanofluids: Experiment and theory. Phys Rev E 2007;76:061203. doi:10.1103/PhysRevE.76.061203.

[54] Rezwan K, Meier LP, Rezwan M, Vörös J, Textor M, Gauckler LJ. Bovine serum albumin adsorption onto colloidal $\mathrm{Al} 2 \mathrm{O} 3$ particles: a new model based on zeta potential and UVvis measurements. Langmuir 2004;20:10055-61. doi:10.1021/la048459k. 


\section{Figure Legends}

Figure 1. Schematic of the redistribution of mobile surface charges and the recharging process for an oil droplet under the externally applied electric field.

Figure 2. Schematic of a spherical oil droplet in a bulk aqueous solution under a uniform DC electric field and the spherical coordinate system.

Figure 3. Schematic of charge distribution in electric double layer near a flat surface in contact with electrolyte and the coordinate system.

Figure 4. Zeta potential distribution along the surface of an oil droplet of $10 \mu \mathrm{m}$ in radius for two different initial zeta potentials, under $E_{\infty}=20 \mathrm{~V} / \mathrm{cm}$.

Figure 5. Zeta potential distribution around oil droplets of different radius for $E_{\infty}=20 \mathrm{~V} / \mathrm{cm}$ and $\zeta_{0}=-50 \mathrm{mV}$.

Figure 6. Zeta potential distribution around an oil droplet of $5 \mu \mathrm{m}$ in radius with an initial zeta potential $\zeta_{0}=-50 \mathrm{mV}$ under different applied electric fields.

Figure 7. Schematic of the model of an oil droplet partially covered by the redistributed mobile surface charges in externally applied electric field. Because of the symmetry, only a half of the spherical droplet is shown here. $\theta$ is the angular variable. $\theta 1$ indicates the boundary position of the surface area covered with surface charges.

Figure 8. The variation of $\theta 1$ (the boundary of the surface area covered with mobile surface charges) with the externally applied electric field $E_{\infty}$ for initial zeta potential $\zeta_{0}=$ $60 \mathrm{mV}$ and drop radius $a=0.6 \mathrm{~mm}$.

Figure 9. Schematics of the distribution of positively charged nanoparticles at the oil-water interface (a) before and (b) after applying electrical field.

Figure 10. The redistribution of positively charged aluminum nanoparticles on the surface of an oil drop of $0.6 \mathrm{~mm}$ in radius under $\mathrm{E}=10 \mathrm{~V} / \mathrm{cm}$ (from left to right) at different time. (a) $t=0 \mathrm{~min}$; (b) $t=3 \mathrm{~min}$; (c) $t=6 \mathrm{~min}$; (d) $t=9 \mathrm{~min}$; (e) $t=12 \mathrm{~min}$; (f) $t=$ $15 \mathrm{~min}$. 


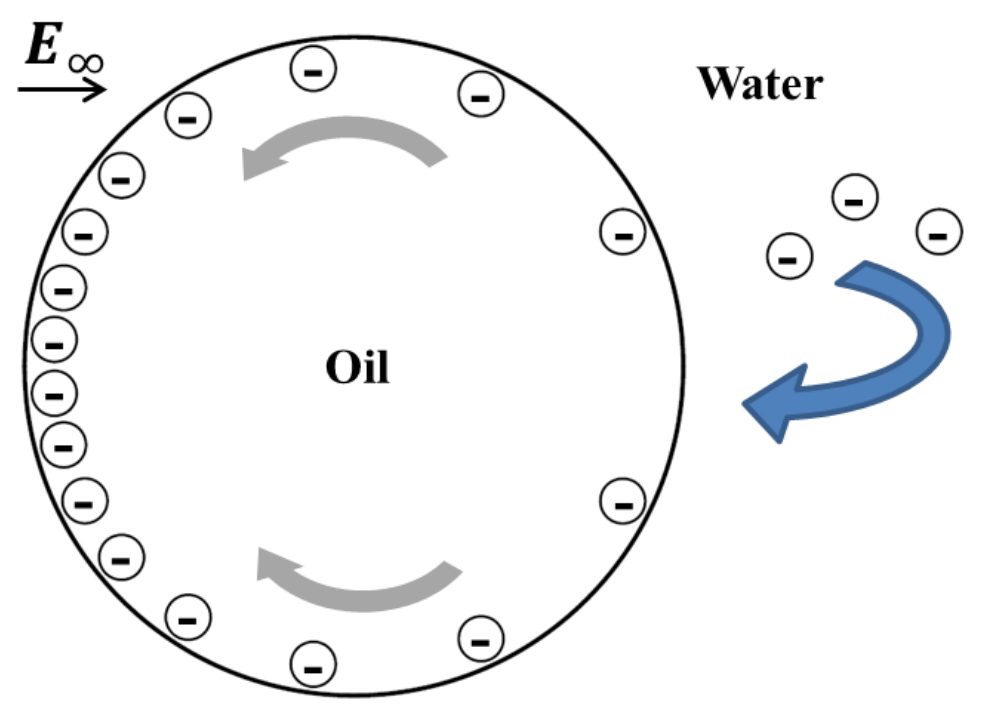

Figure 1 


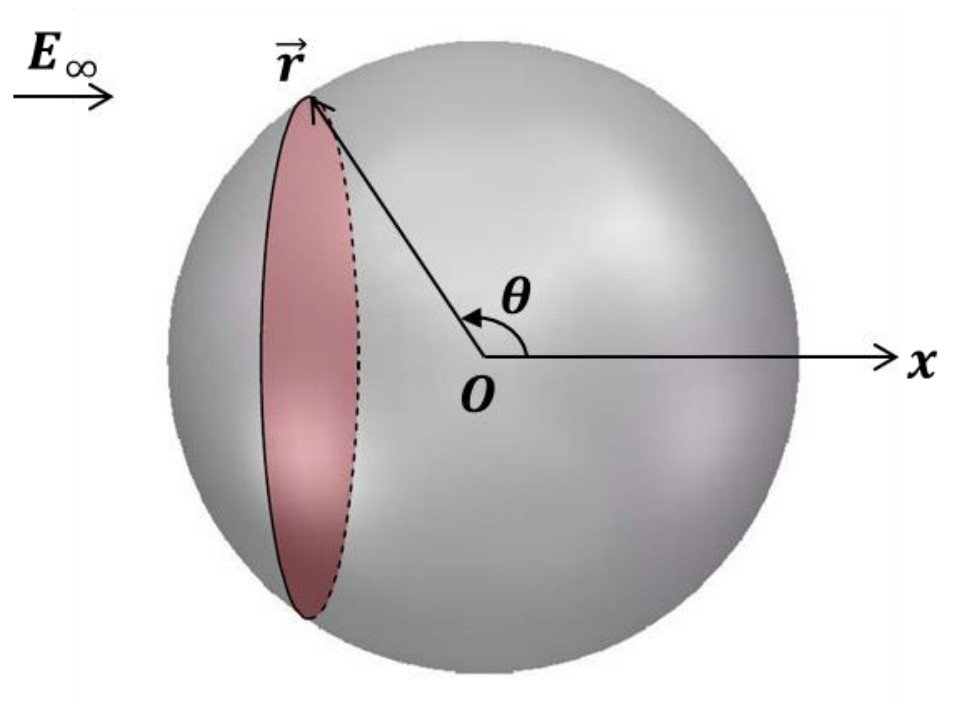

Figure 2 


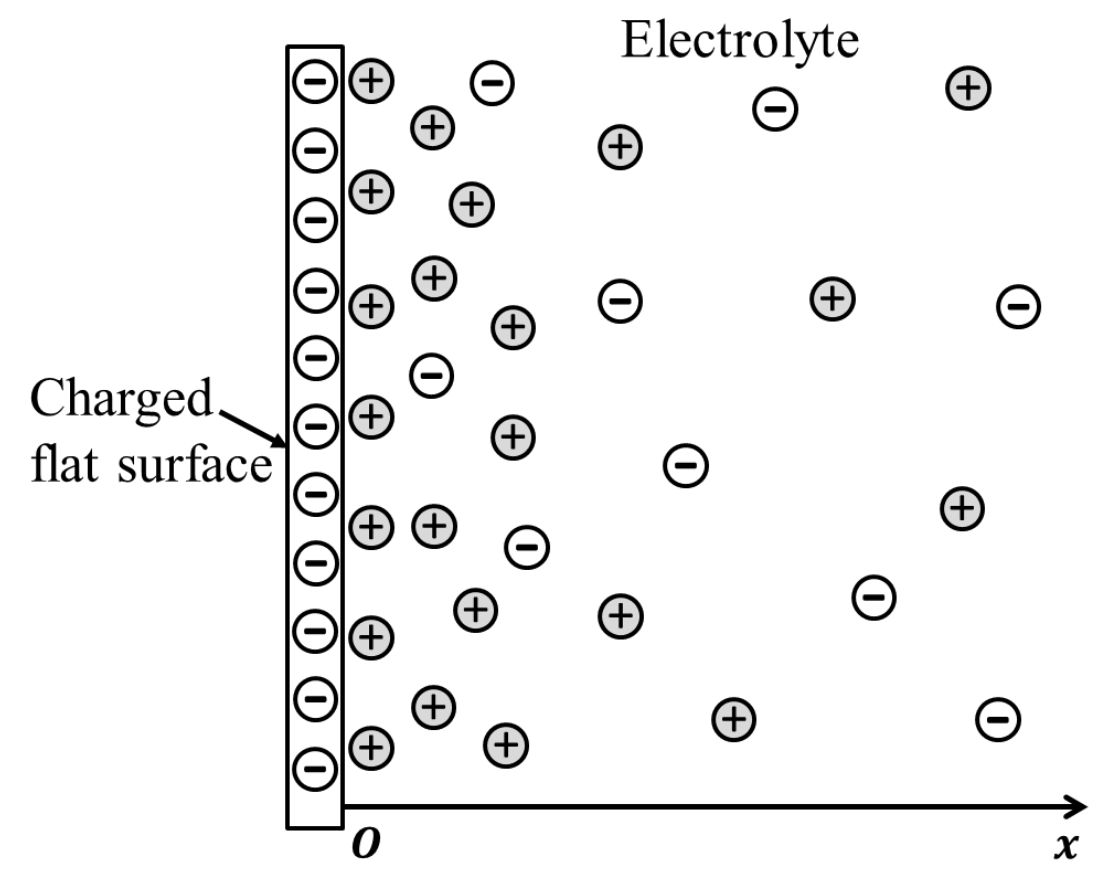

Figure 3 


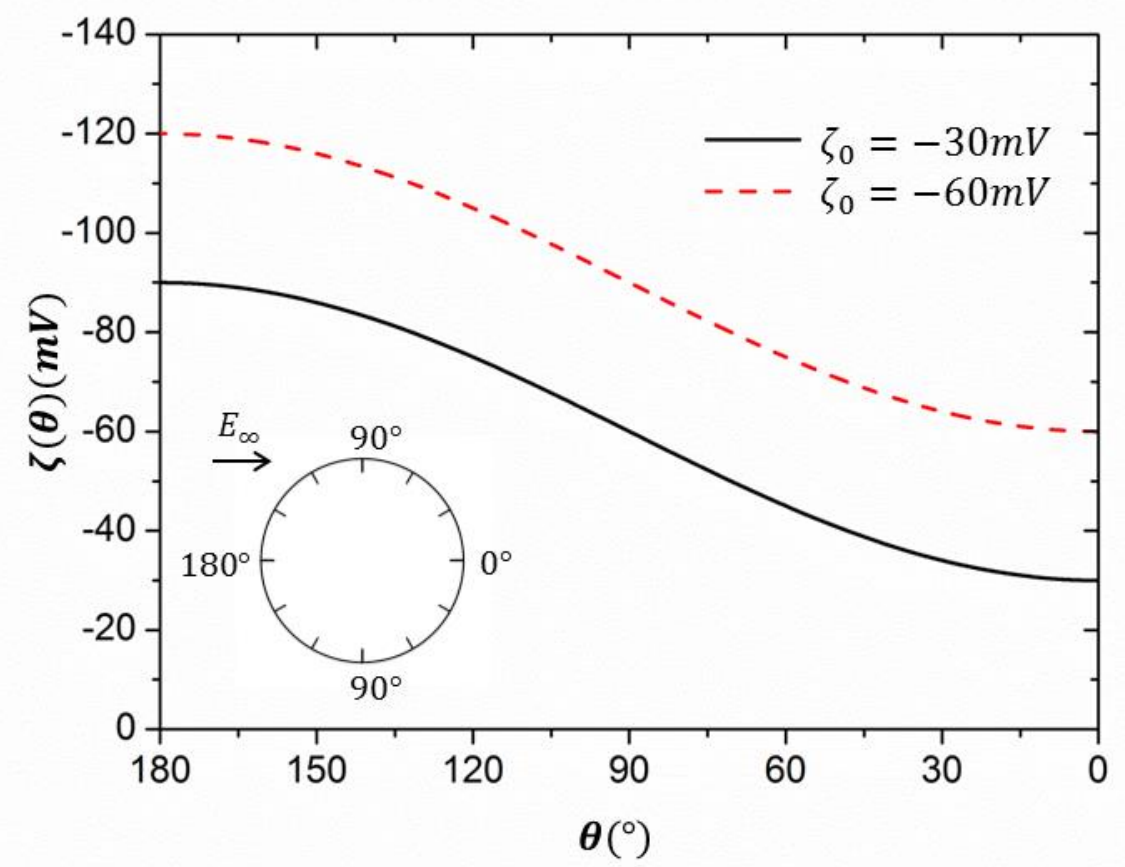

Figure 4 


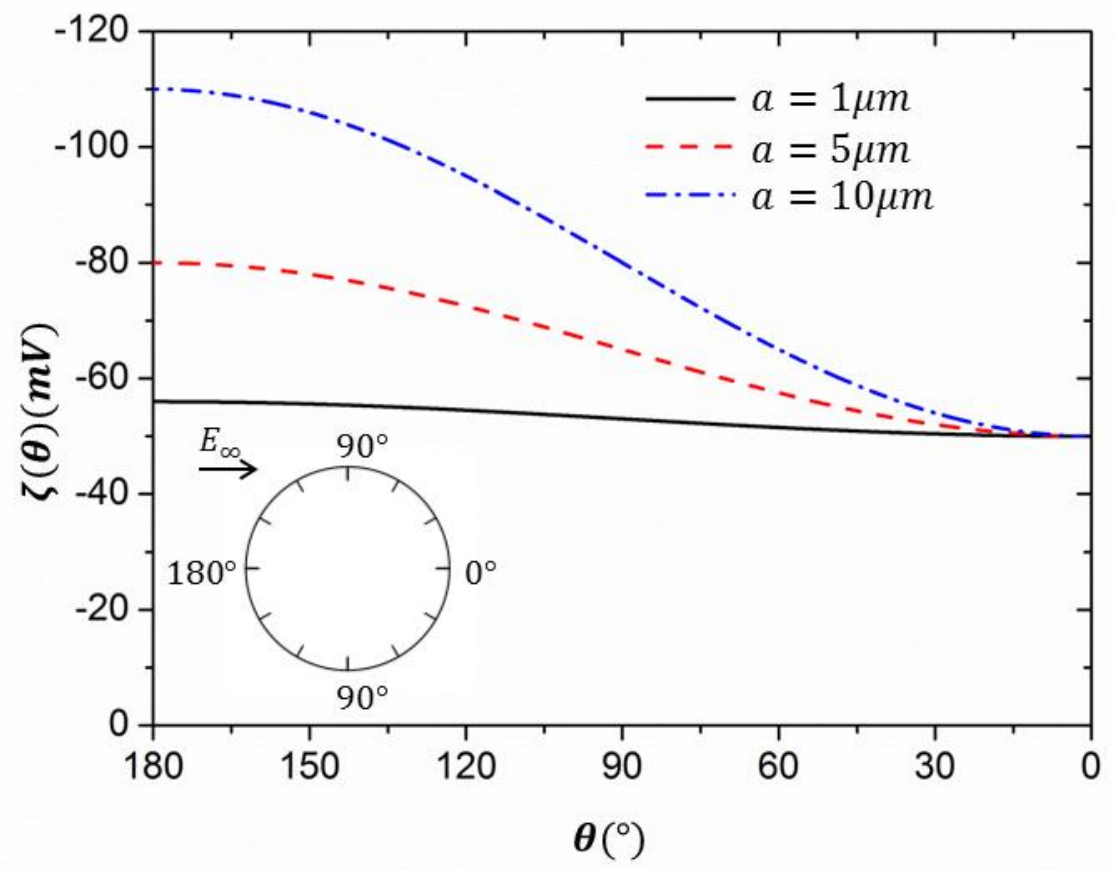

Figure 5 


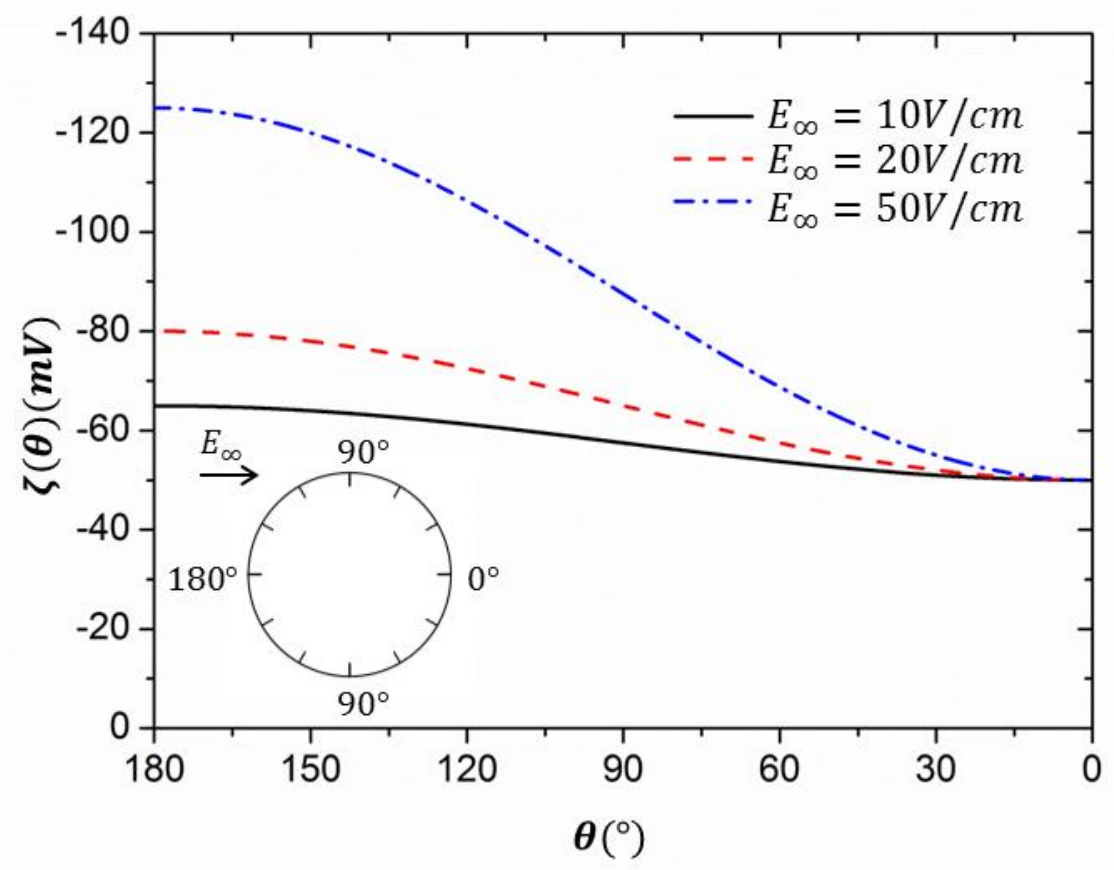

Figure 6 


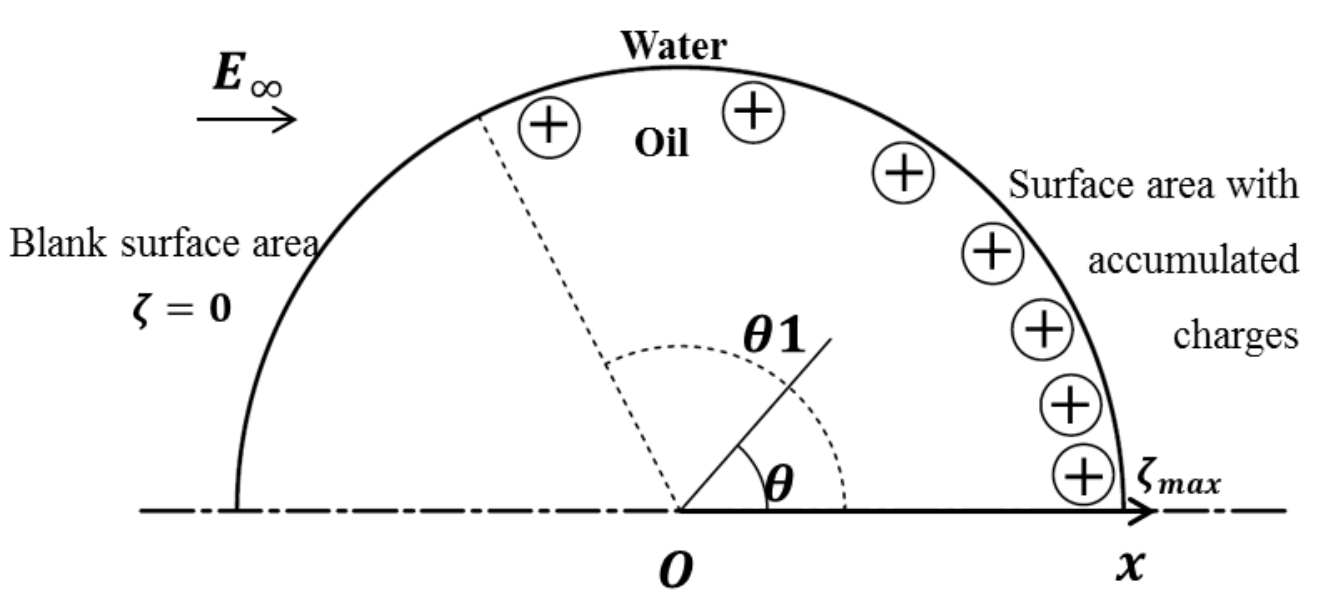

Figure 7 


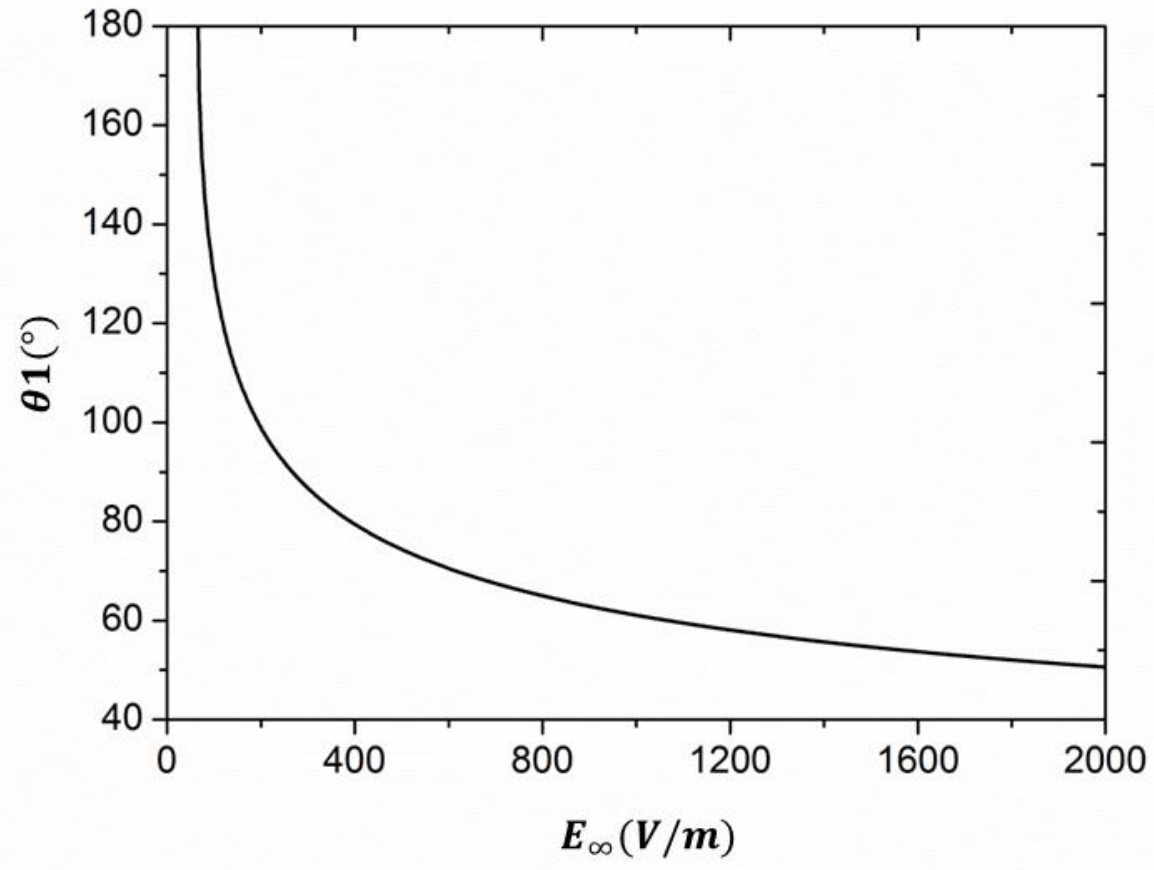

Figure 8 


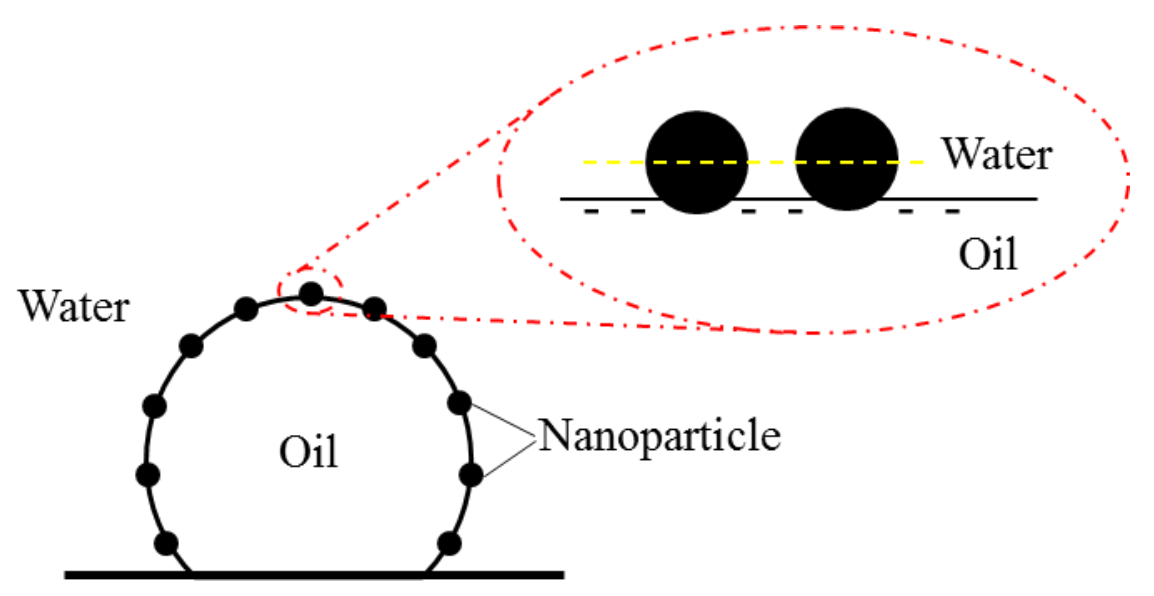

(a)

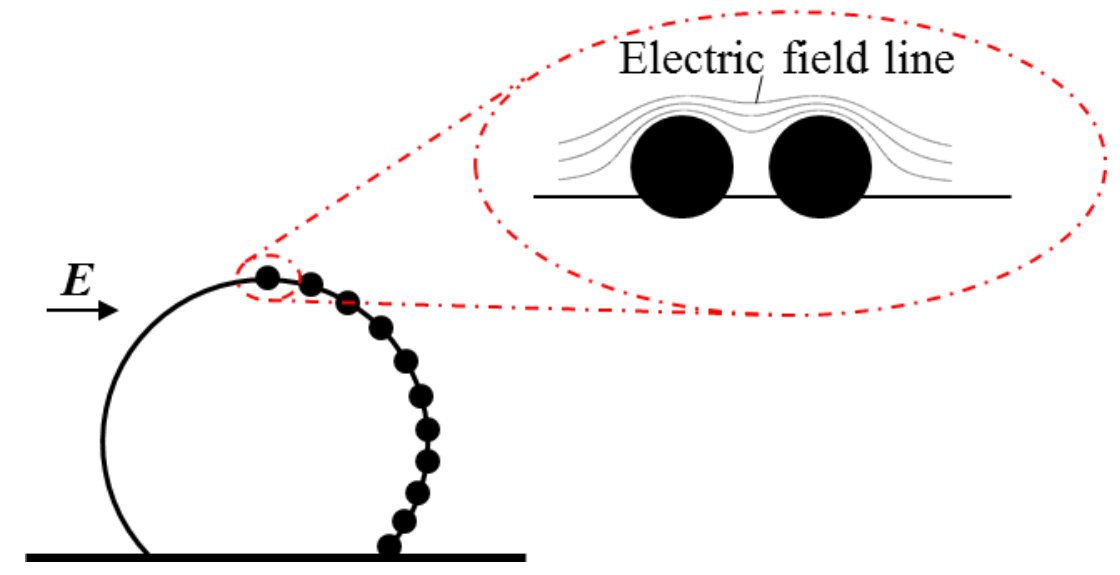

(b)

Figure 9 


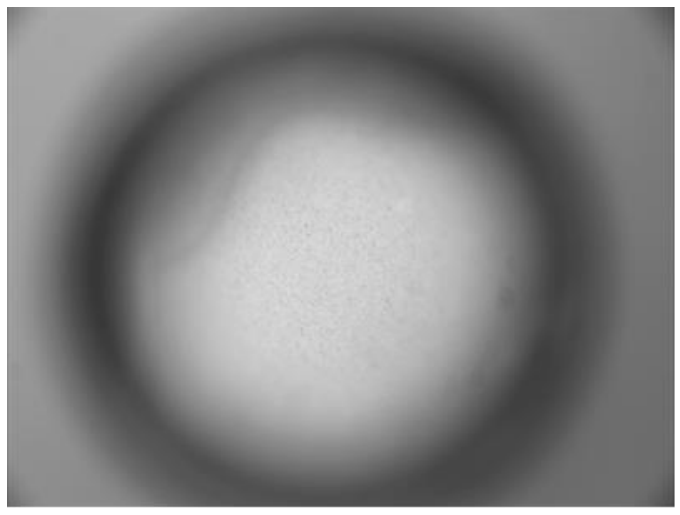

(a)

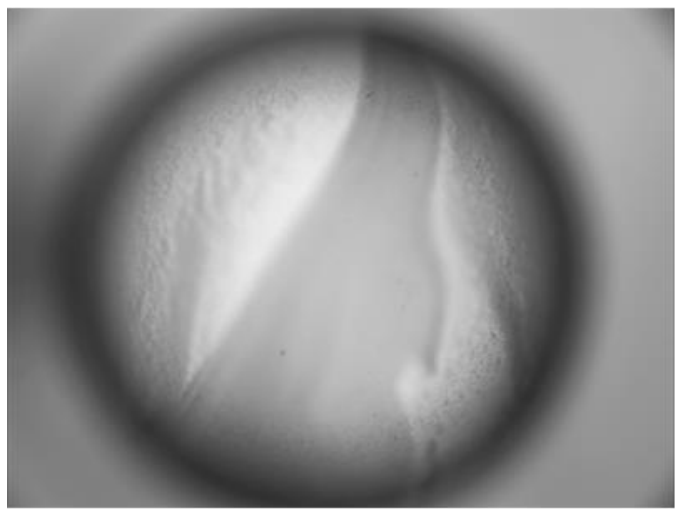

(c)

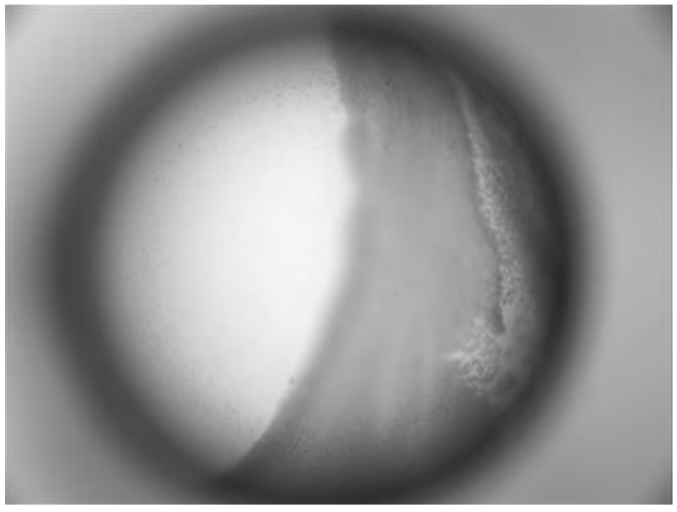

(e)

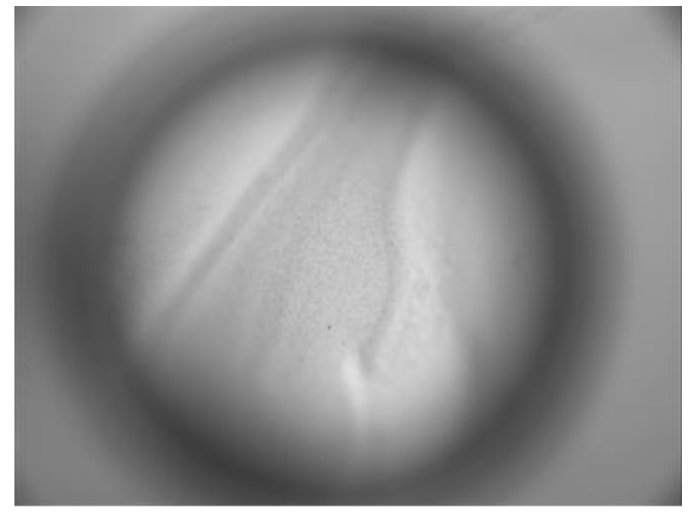

(b)

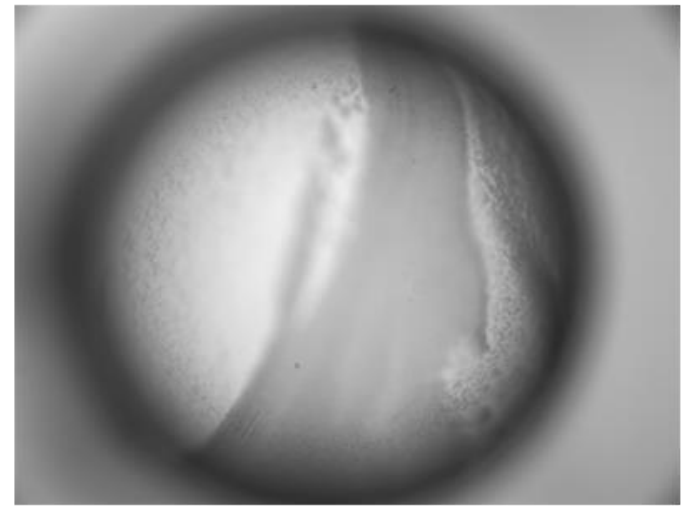

(d)

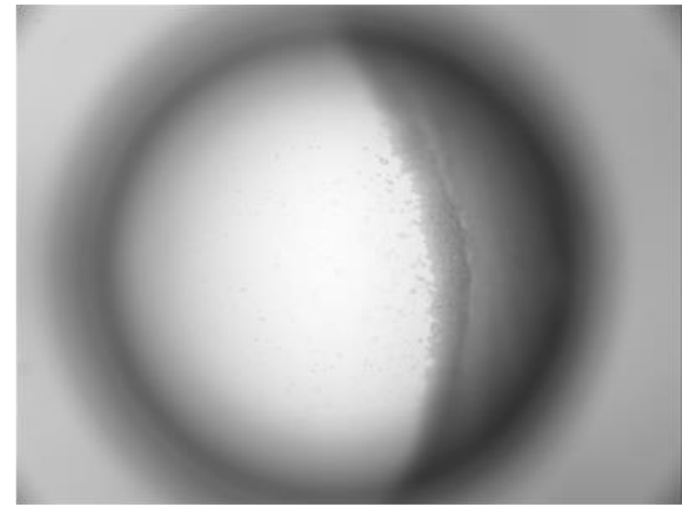

(f)

Figure 10 